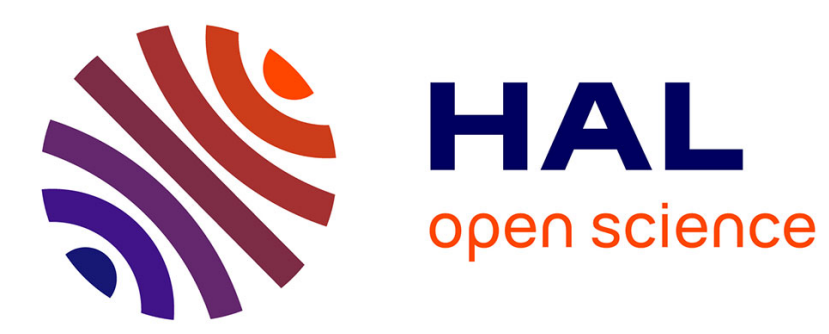

\title{
On the validity of the standard SPICE model of the diode for simulation in power electronics
}

Nouri Massmoudi, Djébé M’Baïri, Bruno Allard, Hervé Morel

\section{To cite this version:}

Nouri Massmoudi, Djébé M'Baïri, Bruno Allard, Hervé Morel. On the validity of the standard SPICE model of the diode for simulation in power electronics. IEEE Transactions on Industrial Electronics, 2001, 48 (4), pp.864-867. hal-00140095

\section{HAL Id: hal-00140095 \\ https://hal.science/hal-00140095}

Submitted on 6 Apr 2007

HAL is a multi-disciplinary open access archive for the deposit and dissemination of scientific research documents, whether they are published or not. The documents may come from teaching and research institutions in France or abroad, or from public or private research centers.
L'archive ouverte pluridisciplinaire HAL, est destinée au dépôt et à la diffusion de documents scientifiques de niveau recherche, publiés ou non, émanant des établissements d'enseignement et de recherche français ou étrangers, des laboratoires publics ou privés. 


\section{Letters to the Editor}

\section{On the Validity of the Standard SPICE Model of the Diode for Simulation in Power Electronics}

Nouri Massmoudi, Djébé M’baïri, Bruno Allard, and Hervé Morel

Abstract-A systematic study of the standard SPICE model of the diode in the case of simulations of power diodes in power electronic applications shows the limits of accuracy with respect to experiments. Therefore, the interest in such a model in power electronic applications is comparable to the high-low resistance model.

Index Terms-p-i-n diodes, power electronics, SPICE model.

\section{INTRODUCTION}

Physics-based models of the power p-i-n diode [2]-[5] are available only in a few circuit simulators. However, the standard SPICE model of the p-n junction is available in all the SPICE-like circuit simulators. This letter covers a systematic study of the validity of the standard SPICE model of the diode in the case of simulations of power diodes in power electronic applications. Simulations in power electronics are often performed using high-low resistor models. It is well known that such a model is not satisfying for power electronic applications, because the structure of such diodes differs form the structure of low-power diodes [1]. However, no systematic study of the model practical validity is available in the literature.

\section{WORKBENCH FOR DIODE TURN-OFF MEASURMENTS}

Obviously, simulations need to be compared with measurements. In most power electronic applications, power diodes are submitted to turn-off and turn-on phases under inductive load switching conditions. It is well known that the turn-off of the power diode is very difficult to estimate by means of simulation [6]. Therefore, we focus on the measurement of the power diode turn-off. The circuit in Fig. 1 enables efficient measurements of the power diode turn-off under inductive load conditions. The circuit design meets two main constraints. First, the diode losses must be limited. Second, the turn-off conditions must be accurately controlled in terms of forward current and reverse applied voltage. The applied reverse voltage $V_{R}$ is controlled by a first power supply in voltage mode. The forward current $I_{F}$ is controlled by a second power supply in current mode. To avoid the self-heating of the diode, the current coming from the current source has to flow through another path most of the time. An insulated gate bipolar transistor (IGBT) Q (IRGPH40F) and a MOSFET M (IRF 740) are used in place of a single switch to bypass the current most of the time. The inductors $L_{I}$ and $L_{Q}$ (Fig. 1) act as current sources during the IGBT and the MOSFET switching phases, respectively. Inductor $L_{I}$ is a large iron inductor and $L_{Q}$ is an air inductor that features high-frequency ratings. The large IGBT enables support of the conduction most of the

Manuscript received January 12, 2001; revised February 1, 2001. Abstract published on the Internet June 6, 2001.

N. Massmoudi and D. M'baïri are with LEII, Département de Génie Electrique, École Nationale d'Ingenieurs de Sfax, BPW 3038 Sfax, Tunisia.

B. Allard and H. Morel are with CEGELY CNRS UPRESA 5005, INSA de Lyon, 69621 Villeurbanne Cedex, France (e-mail: Herve.Morel@cegely.insalyon.fr).

Publisher Item Identifier S 0278-0046(01)06289-X. time (Fig. 2). The MOSFET is turned on before the IGBT is turned off Then, the MOSFET turns off and the diode becomes forward biased for a short time. Finally, using such a technique, the diode is associated to the fast switch $M$ during its turn-off, without any significant self-heating.

The current flowing through the diode $i_{D}$ is measured with an aselfic shunt (by T\&M Research) of 1.2-GHz bandwidth, placed in series with the diode anode. The voltage drop across the diode is obtained by computing the difference between the anode and cathode voltage values

$$
v_{D}=v_{A}-v_{C}
$$

where $v_{A}$ and $v_{C}$ are the anode and cathode voltages obtained by $500-\mathrm{MHz}$ voltage probes (Tek P6139A), placed at the diode pins nearest to the diode case. This technique enables reduction of the parasitic stray inductance of the diode pins. The measured waveforms $i_{D}(t)$ and $v_{D}(t)$ are obtained with an oscilloscope (Tek TDS744A) Then, they are analyzed to obtain the main transient parameters, like $i_{R M}, v_{R M}, t_{R R}, d i_{F} / d t$, and $d i_{R} / d t$, as shown in Fig. 3.

\section{EXTRACTION OF THE PARAMETERS OF THE DiODE SPICE MODEL}

We have chosen to fit the model parameters with the turn-off conditions to obtain the best simulation results. The proposed estimation procedure is based on the comparison of the transient waveforms $i_{D}(t)$ and $v_{D}(t)$ during the diode turn-off, between measurements (obtained in Section II) and simulation results using the SPICE model. The curve fitting addresses only the turn-off phase.

The role of each parameter with respect to the turn-off phase is analyzed before the discussion of the parameter estimation procedure.

\section{A. Role of the Circuit Parameters}

The Kirchhoff's voltage law for the loop comprising the source $v_{R}$, the diode, and the MOS (Fig. 1) yields

$$
\frac{d i_{D}}{d t}=-\frac{v_{R}-v_{D S}-v_{D}-r_{s} i_{D}}{L_{m}} .
$$

The first step is to fit the simulated current to the measured current before the reverse voltage becomes negative. In this phase, the diode voltage is very low due to the on state. Therefore, $v_{D}$ and $r_{S} i_{D}$ are negligible with respect to $V_{R}$. Hence, the forward current $i_{F}$, the MOSFET drive $R_{G}^{M}$, and the rise time of the controlled signal $v_{G}^{M}$ have to be fitted to obtain a good agreement on the current waveform. Obviously, the parasitic inductance $L_{m}$ has to be estimated carefully since it controls the current rate to some extent. In this case, $L_{m} \sim 80 \mathrm{nH}$.

The circuit parameters are estimated only once. The values are kept unchanged, regardless of the diode under test and the operating conditions ( $I F$ and $V R$ ).

\section{B. Parameters of the Diode SPICE Model}

The standard SPICE model of the diode is given in [7]. This model represents a $\mathrm{p}-\mathrm{n}$ junction. The space-charge region is represented by the junction capacitance $C_{J}$

$$
C_{J}=\frac{C J 0}{\left(1-\frac{v_{D}}{V J}\right)^{M}}
$$




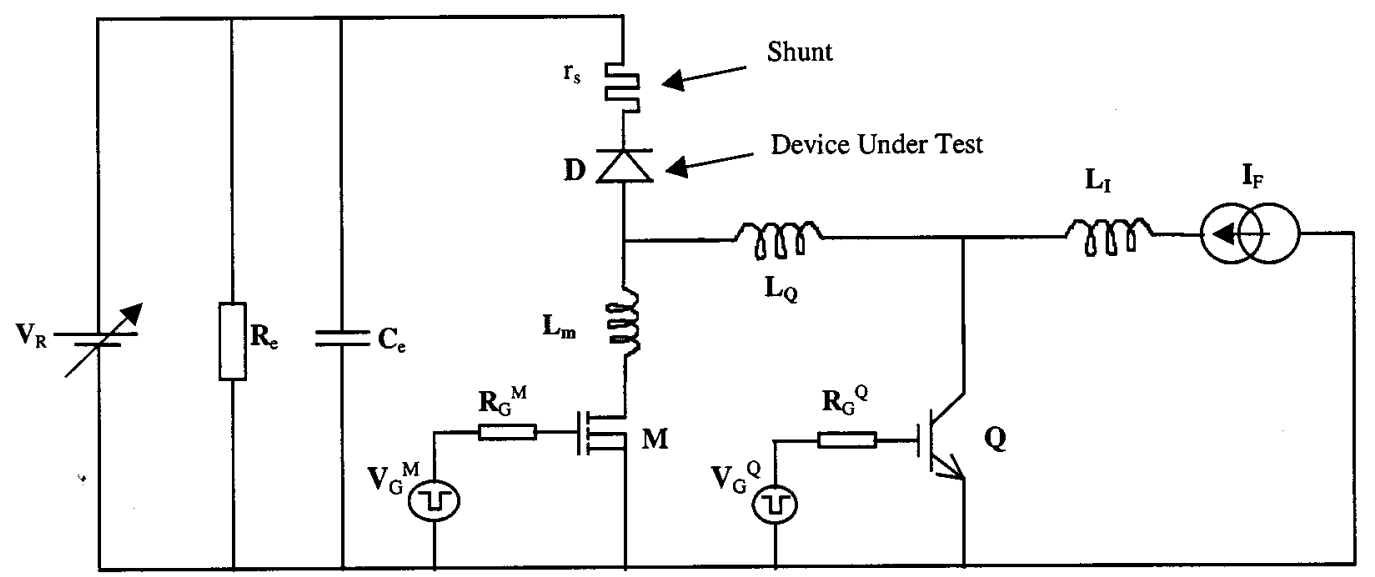

Fig. 1. Circuit for power diode measurements in transient conditions.
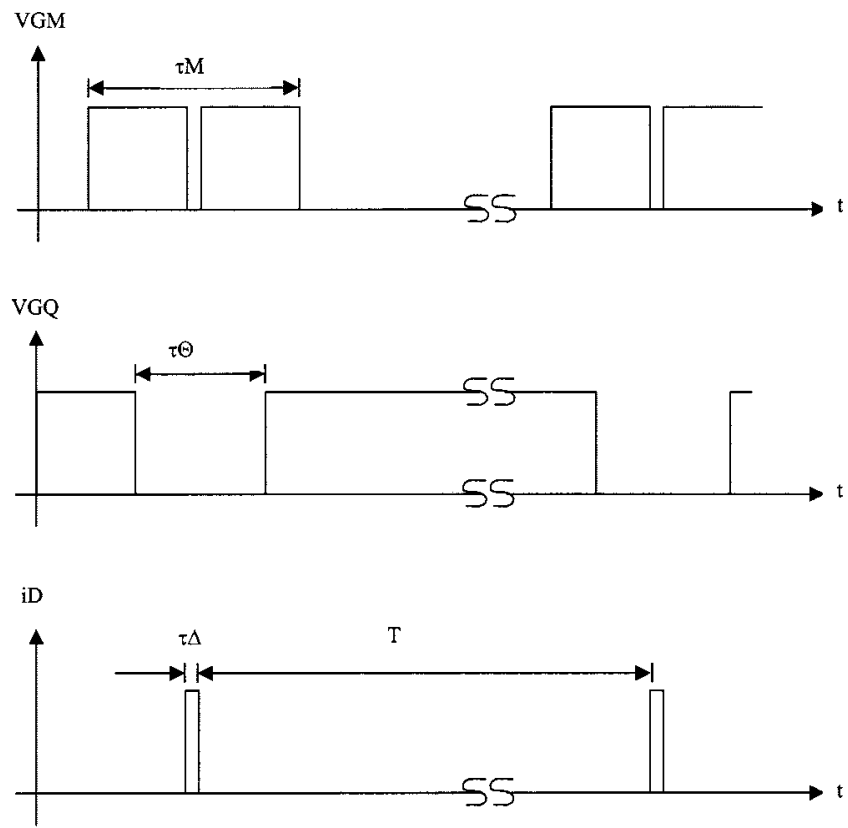

Fig. 2. Typical driven signal and diode current waveforms. $\tau_{M} \sim 300 \mu \mathrm{s}$ is the conduction time of the MOSFET. $\tau_{Q} \sim 200 \mu \mathrm{s}$ is the off-state time of the IGBT. $\tau_{D}$ is the conducting time of the diode under test $\left(2 \mu \mathrm{s}<\tau_{D}<10 \mu \mathrm{s}\right)$.

where $C J 0, V J$, and $M$ are classical model parameters. The minority carrier storage is modeled by the charge computed as $T T \times I_{D}$ where $T T$ is the transit time (the minority carrier lifetime) and $I_{D}$ is the current in static condition, given by the classical relation

$$
I_{D}=I S\left(\exp \frac{v_{D}}{N u_{T}}-1\right)
$$

where $I S$ is the saturation current, $N$ is the emission coefficient, and $u_{T}=k T / q$ is the thermal potential.

The first two parameters, $I S$ and $N$, are estimated from the static characteristic of the device. Our systematic analysis of the role of the model parameters on the turn-off behavior has shown that the three main parameters are $T T, C J 0$, and $M$. The other parameters do not act significantly on the turn-off behavior if acceptable values of the parameters are used with respect to their physical meaning. For instance, the serial resistance $R S$ acts on the damping behavior of the

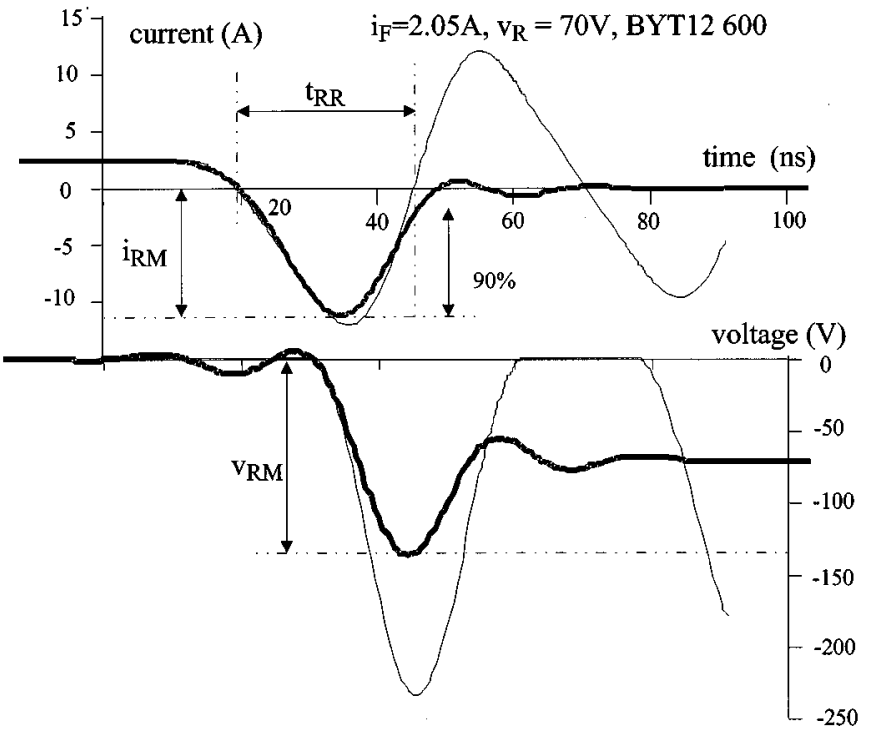

Fig. 3. The thin curve corresponds to the simulation of the BYT12 600 turn-off for the operating condition: $i_{F}=2.05 \mathrm{~A}, v_{R}=70 \mathrm{~V}$. The bold curve corresponds to measurements at the same conditions. Main transient parameters describing the p-i-n diode turn-off are superimposed.

turn-off, but only for very large values that are not relevant to the physical knowledge nor the static characteristic. Obviously, the breakdown voltage parameters $B V$ and $I B V$ are set in agreement with the device characteristics.

The simulation circuit is given in Fig. 4

In the following, the set of optimal parameter values corresponds to the BYT12p600 power diode.

The parameter $T T$ acts on the minority carrier storage inside the $\mathrm{n}$-neutral region of the $\mathrm{p}^{+} \mathrm{n}$ junction. However, the physical phenomenon in the low doping region of a power $p$-i-n diode is classically the high-level injection characterized by the ambipolar lifetime, but this phenomenon is not modeled in the standard SPICE model. Therefore, a common idea is to modify the value of the parameter $T T$ to obtain the behavior of the p-i-n diode. Fig. 5 shows the influence of parameter $T T$ on the turn-off waveforms. The values $I_{R M}$ and $t_{R R}$ increase weakly and $V R M$ decreases for increasing values of the zero-bias capacitance $C J 0$. Fig. 6 shows that the transient parameters $i_{R M}$ and $t_{R R}$ decrease and $V_{R M}$ increases highly for increasing values of the grading coefficient $M$. 


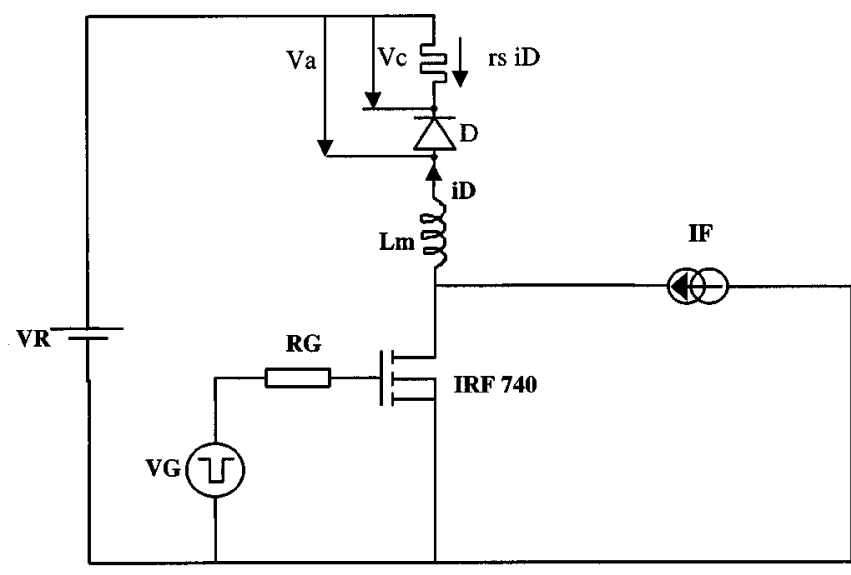

Fig. 4. Principal circuit for the measurements. This is also the simulation circuit.

current $i_{D}(A)$

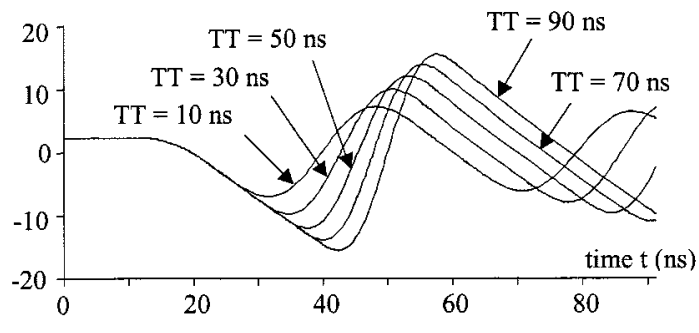

voltage $v_{n}(V)$

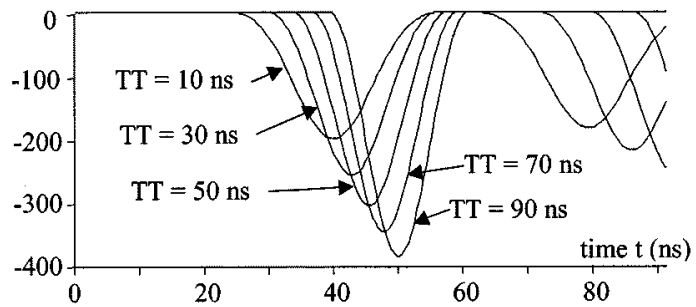

Fig. 5. Influence of parameter $T T$ on the diode turn-off obtained by simulation of the standard SPICE model of the diode. The base parameter set is the optimal set for the BYT12 600 (Fig. 3).

\section{ANALYZING THE “OPTIMAL” CASE}

It appears that a correct fitting of both curves $i D(t)$ and $v D(t)$ is impossible. Therefore, we have chosen to perform a fitting that enables the simulation to estimate correctly the current waveform. A set of "optimal" parameter values may be obtained by fitting experimental data. The optimization criterion is the sum of relative error on $I_{R M}$ and $t_{R R}$. Fig. 3 shows the comparison between measurements achieved on a BYT12 600 diode in the case where $i_{F}=2.05 \mathrm{~A}$ and $v_{R}=70 \mathrm{~V}$, and the corresponding simulation for the optimal values of the parameters $T T=40 \mathrm{~ns}, C J 0=2.0 \mathrm{nF}$, and $M=0.3$. Moreover, some parameters have been set to nondefault values: $R S=30 \mathrm{~m} \Omega, I S=10^{-13}$ $\mathrm{A}, B V=600 \mathrm{~V}$, and $I B V=10^{-13} \mathrm{~A}$.

Fig. 3 shows the agreement between measurement and simulation for the first reverse peak in the current waveform. The agreement for the values of $i_{R M}$ and $t_{R R}$ is very good. However, the agreement on the reverse voltage peak is not good and the error on the transient parameter $v_{R M}$ is about $66 \%$. Unfortunately, no set of parameter values enables reduction of the latter error. Moreover, the damping behavior of the experimental curve is not correctly estimated by the simulation. Indeed,

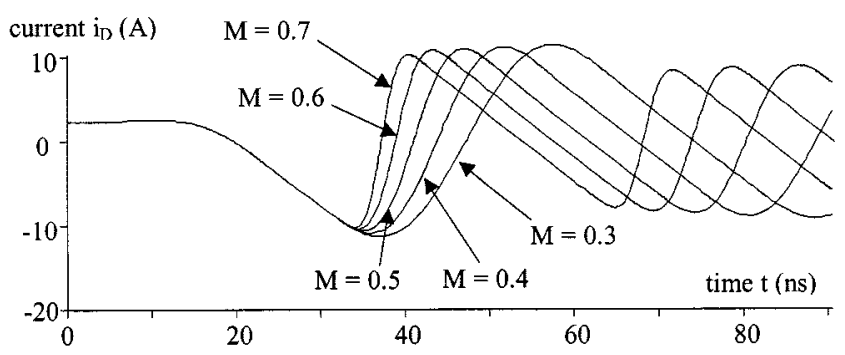

voltage $v_{D}(V)$

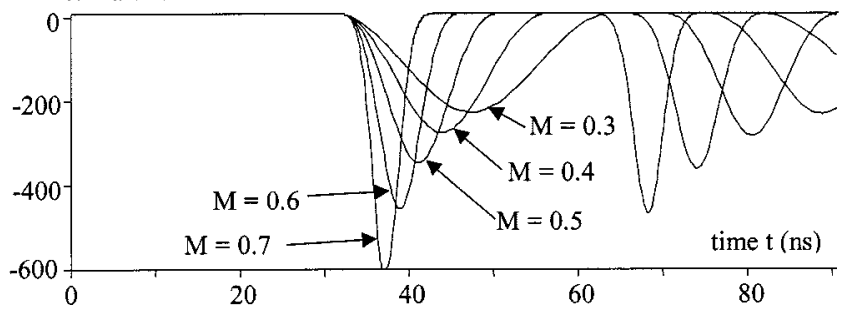

Fig. 6. Influence of parameter $M$ on the diode turn-off obtained by simulation of the standard SPICE model of the diode. The base parameter set is the optimal set for the BYT12 600 (Fig. 3).

whatever the values of the parameters $T T, C J 0$, and $M$, we have never found such a damping behavior.

\section{ON THE VALIdity Domain of THE Standard SPICE Model}

We have just shown that it is possible to identify values of the SPICE model parameters from a fitting with a diode turn-off for $i_{R M}$ and $t_{R R}$ transient parameters. The obtained results are not completely satisfying, but they give quite good results regarding the estimation of the current waveform. We have tried also to study whether the simulation continues to give partially acceptable results in the case of other experimental conditions. Indeed, the agreement is no longer acceptable, even in the case of the current waveform.

\section{CONCLUSION}

We have developed a workbench for the characterization of the diode-switching behavior inside a power electronic application. The workbench enables a systematic measurement of the diode turn-off for a large range of forward currents and reverse applied voltages.

Then, an identification procedure of the parameters of the standard SPICE model of the p-n junction was proposed. It has been shown that, for a given operating condition, good results may be obtained for the current waveform. However, the results are not satisfactory for the voltage waveform. Moreover, the agreement is bad for any operating condition different from the identification case.

Finally, the standard SPICE model is not useful for the simulation of power electronic applications. Therefore, the usefulness of the diode SPICE model is comparable to the high-low resistance model.

Our conclusion does not differ from common knowledge, but the main results are to be found in the systematic study of the diode SPICE model.

\section{REFERENCES}

[1] N. Mohan, T. M. Undeland, and W. P. Robbins, Power Electronics. New York: Wiley, 1995.

[2] P. O. Lauritzen and C. L. Ma, "A simple diode model with reverse recovery," IEEE Trans. Power Electron., vol. 6, pp. 188-191, Apr. 1991.

[3] H. Morel, S. H. Gamal, and J. P. Chante, "State variable modeling of the power PIN diode using an explicit approximation of semiconductor device equations: A novel approach," IEEE Trans. Power Electron., vol. 9, pp. 113-120, Jan. 1994. 
[4] A. G. M. Strollo, "A new SPICE model of power P-I-N diode based on asymptotic waveform evaluation," IEEE Trans. Power Electron., vol. 12, pp. 12-20, Jan. 1997.

[5] H. A. Mantooth, "A unified diode model for circuit simulation," IEEE Trans. Power Electron., vol. 12, pp. 816-823, Sept. 1997.

[6] F. Bertz, "A simplified theory of the p-i-n diode," Solid-State Electron., vol. 20, pp. 709-714, 1977.

[7] G. Massobrio and P. Antogneti, Semiconductor Device Modeling With SPICE. New York: McGraw-Hill, 1993.

\section{A Novel Torque-Ripple Reduction Strategy for Direct Torque Control}

\author{
D. Telford, M. W. Dunnigan, and B. W. Williams
}

\begin{abstract}
This letter presents a simple duty-cycle control scheme for direct torque control of an induction motor. The scheme reduces torque ripple, controls the average output torque, and reduces the variation in switching frequency. The effectiveness of the proposed technique is demonstrated through experimental results.
\end{abstract}

Index Terms-Direct torque control, switching frequency, torque ripple.

\section{INTRODUCTION}

Conventional direct torque control (DTC) has the drawbacks of high torque ripple, variable switching frequency, and the mean torque output not matching the torque demand [1]. Several papers propose methods for overcoming some of these problems [1]-[4]. However, the techniques either erode the simplicity of DTC by utilizing a machine model to predict the rate of change of torque, or require high sampling rates in order to maintain effective control.

This letter presents a simple duty-cycle control scheme that controls the average torque to be equal to the lower hysteresis limit using a duty-cycle controller. Analysis of the nonlinear effects in DTC, supported by experimental results, demonstrates that this technique reduces torque ripple, particularly in the low-speed region, controls the mean output torque, and reduces the variation in switching frequency. A list of symbols used in this letter is given in Table I.

\section{FluX AND TORQue CONTROL WITH DTC}

The equation for electromagnetic torque in an induction machine can be written as

$$
T_{e}=\frac{3}{2} P \frac{L_{m}}{\sigma L_{s} L_{r}}\left|\bar{\psi}_{r}\right|\left|\bar{\psi}_{s}\right| \sin (\gamma) .
$$

In DTC, voltage-vector selection is limited to the eight available from the inverter including the two zero voltage vectors. The stator flux magnitude can be controlled by selecting one of the available voltage vectors that will move the flux in the desired direction. Torque can be increased (decreased) by selecting a voltage vector which will increase (decrease) the magnitude of the quadrature component of the stator flux relative to the rotor flux. As the selected voltage vector is applied for

Manuscript received December 20, 2000; revised February 1, 2001. Abstract published on the Internet June 6, 2001.

The authors are with the Department of Computing and Electrical Engineering, Heriot-Watt University, Edinburgh, EH14 4AS, U.K. (e-mail: telfordd@cee.hw.ac.uk).

Publisher Item Identifier S 0278-0046(01)06290-6. the whole of the sampling period, then the resulting increase in torque can be significant, giving a large torque ripple. It is proposed that a duty-cycle control scheme is implemented to reduce the torque-ripple magnitude.

\section{EFFECT OF NON-LineARITIES ON RATE OF CHANGE OF TORQUE}

The change of torque over an arbitrary time period $t$ is given as

$$
\begin{aligned}
& d T_{e}=\frac{3}{2} P \frac{L_{m}}{\sigma L_{s} L_{r}}\left|\bar{\psi}_{r}\right|\left|\bar{\psi}_{s}\right| \\
& \cdot\left(\sin \left(\gamma\left(t_{0}\right)+\frac{d \gamma}{d t} t\right)-\sin \left(\gamma\left(t_{0}\right)\right)\right)
\end{aligned}
$$

where (in the stator-flux-oriented reference frame)

$$
\frac{d \gamma}{d t}=\omega_{e s}-\omega_{e r} \quad \text { and } \quad \omega_{e s}=\frac{v_{q s e}-R_{s} i_{q s e}}{\psi_{d s e}} .
$$

In DTC, the flux plane is divided into six $60^{\circ}$ sectors allowing selection of the appropriate voltage vector. Assuming that, in the steady state, the hysteresis limits are set to reduce torque with a zero voltage vector, then only two active voltage-vector selections are possible, increasing torque/increasing flux, or increasing torque/decreasing flux. Fig. 1(a) shows the variation in $v_{q s e}$ for each possible voltage vector as the flux position varies in two flux sectors. The effect of this voltage variation on the rate of torque increase can be calculated from (2) and (3). Fig. 1(b) shows the possible maximum and minimum rate of change of torque when a voltage vector is applied and the decrease of torque when a zero voltage vector is applied. This is based on a torque angle of $17^{\circ}$, which gives rated torque for the test machine, an applied voltage magnitude of $300 \mathrm{~V}$, and $100 \%$ duty cycle (as in conventional DTC). From Fig. 1(b), the increase of torque is significantly higher than the decrease of torque, particularly at low frequencies, resulting in a high torque ripple and reduced switching frequency.

To address this problem, it is proposed that a duty-cycle control scheme is implemented which controls the average torque increase when a voltage vector is applied to match the torque decrease when a zero voltage vector is applied. Using this method, Fig. 1(c) shows the possible maximum and minimum rate of rise of torque when a voltage vector is applied and the rate of decrease of torque when a zero voltage vector is applied. The scheme reduces the rate of increase of torque, particularly at low frequencies. The variation in torque increase is also reduced.

\section{Duty Cycle Control Scheme}

Consider operation of DTC where the scheme is used to control the average torque to be equal to the lower hysteresis limit and the torque is allowed to decay naturally. If the duty cycle is correct and the average torque increase matches the average torque decrease, then the mean torque matches the lower hysteresis limit. If the duty cycle is too long, then the average torque increase will be higher than the average torque decrease, and the mean torque will become higher than the hysteresis limit. If the duty cycle is too short, then the average torque increase will be too low, and the mean torque will become lower than the hysteresis limit. By varying the duty cycle to control the average output torque to be equal to the lower hysteresis limit, the conditions for reduced torque ripple will be met. The scheme can be implemented by adding a simple duty-cycle control system to the DTC architecture. The lower hysteresis limit is set to be equal to the torque reference $T_{\text {eref }}$. The torque error drives an integrator which adjusts the duty cycle. The resulting DTC architecture is shown in Fig. 2. 